\section{O Sistema Único de Saúde, 20 anos: balanço e perspectivas}

\author{
The Unified National Health System, 20 years: \\ assessment and perspectives
}

\author{
${ }^{1}$ Faculdade de Filosofia \\ e Ciências Humanas, \\ Universidade Federal de \\ Minas Gerais, Belo Horizonte, \\ Brasil. \\ Correspondência \\ T. M. G. Menicucci \\ Faculdade de Filosofia \\ e Ciências Humanas, \\ Universidade Federal de \\ Minas Gerais. \\ Rua Groenlândia 77, apto. 401, \\ Belo Horizonte, $M G$ \\ 30320-060, Brasil. \\ telmenicucci@fafich.ufmg.br
}

\section{Abstract}

This article reviews the strides and challenges of the Brazilian Unified National Health System (SUS), based on an assessment of the results achieved and the constraints for effective consolidation of it objectives. The article focuses on three issues: the process of implementing the SUS, highlighting the institutional and political constraints, some of which as effects of previous policies; strides towards more accessible and comprehensive care, with an impact on some health indicators, and institutional innovations with positive impacts on the system's management; and challenges for consolidating the objectives, as a result of exogenous factors in relation to the SUS, emphasizing the need for its politicization and greater legitimization.

Single Health System; Health Public Policy; Health Management

\section{Introdução}

A Constituição Federal de 1988 estabeleceu os princípios e diretrizes para uma completa reorganização do Sistema de Saúde a partir da formalização de um princípio igualitário e do comprometimento público com a garantia desta igualdade. A criação do Sistema Único de Saúde (SUS) pode ser considerada uma "carta fundadora" de uma nova ordem social no âmbito da saúde, baseada nos princípios da universalidade e igualdade, e organizado sob as diretrizes da descentralização, atendimento integral e participação da comunidade.

Uma das formas de se fazer um balanço do SUS quando se comemoram os seus 20 anos, pode ser fazendo uma avaliação dos resultados alcançados quanto a esses objetivos e quanto à implementação dessas diretrizes e quais os principais desafios para isso. Nessa perspectiva, o artigo enfatiza três ordens de questões inter-relacionadas: o processo de implantação do SUS; os avanços efetivos em relação ao acesso, à integralidade e à gestão; e os desafios decorrentes de fatores exógenos ao SUS.

\section{O processo de implantação do SUS}

Na década de 90 se colocou a tarefa de transformar em realidade os dispositivos definidos na Constituição Federal. Devido às suas enormes di- 
mensões, esse processo tem implicado alto grau de criatividade e inovação, mas, para além das questões institucionais e de gestão em que os avanços são significativos, o processo de implementação do SUS tem sido repleto de ambigüidades, avanços e dificuldades.

Para evitar avaliações simplistas que se limitem a contrapor os objetivos do SUS e os seus resultados, destaco a necessidade de discutir o processo de implementação de políticas públicas, cujo sucesso está associado à capacidade de obtenção de convergência entre os agentes implementadores em torno dos objetivos da política e, particularmente, do suporte político. Sua implantação exige decisão e iniciativa governamental e instrumentos para efetivá-la, entre eles a disponibilidade de recursos financeiros e suporte político organizado, particularmente por parte dos grupos sociais afetados positivamente pela política. A questão da viabilidade política é crucial, dado que sustentação política e legitimidade da política são variáveis fundamentais para a implementação.

Daí a pergunta que é preciso responder: qual a sustentação política e a legitimidade dos princípios definidos para o SUS que permitissem a efetiva universalidade e igualdade? Meu argumento é que em função da fragilidade de suas bases de apoio, mais do que mera tradução prática de decisões, a implantação da reforma se mostrou, de fato, como um processo de formulação da política de saúde, cujo resultado será a consolidação de um sistema híbrido - público e privado -, apesar da definição legal de um sistema único, público, universal e gratuito 1 .

O contexto político nacional e internacional de implantação do SUS não se mostrou favorável. A configuração conservadora dos governos no período da transição democrática estava em perfeita sintonia com o ambiente internacional, marcado pela rediscussão do papel do Estado, que se traduzia em propostas de novos modelos de políticas sociais, com focalização do gasto público nos setores mais pobres. Ao processo de democratização se seguiram os de ajuste e estabilização econômica, acompanhados das reformas estruturais, em sentido inverso à ampliação das atribuições governamentais e dos direitos sociais recém-consagrados na Constituição Federal, em sintonia com o receituário internacional 2.

A conjuntura de crise fiscal, associada ao fortalecimento de posições conservadoras e voltadas para o mercado, colocou constrangimentos à implantação do SUS, limitando a possibilidade de ampliação dos serviços de saúde para garantir a universalização. Ao se traduzir na redução do gasto público, funcionou como freio objetivo e ideológico à atuação redistributiva do Estado.
A heterogeneidade na composição e nos posicionamentos políticos dos integrantes do "movimento sanitário", base social da reforma de saúde, se evidenciou no momento de sua implantação quando se atualizaram as divergências de interesses, que durante o movimento pela redemocratização tinham sido escamoteados em função da agregação em torno da retomada do regime democrático e da reforma sanitária enquanto um projeto ético de caráter coletivo. Particularmente, acirram-se as divergências partidárias em decorrência do fortalecimento das identidades partidárias e aprofunda-se o corporativismo entre o movimento médico que se distancia do projeto da reforma sanitária ${ }^{3}$. Por sua vez, o movimento popular em saúde não foi capaz de garantir o apoio dos usuários dos serviços de saúde. Contribuiu para isso não apenas sua fraca mobilização, mas a característica da reforma, que faz com que os seus benefícios sejam muito dispersos e só perceptíveis a longo prazo, o que fez com que os responsáveis pela reforma não pudessem contar com o apoio mobilizado do público alvo da política de saúde.

A proposta de um sistema de saúde igualitário chocou-se com o legado histórico de uma sociedade marcada pela diferenciação e pela segmentação no próprio campo da atenção à saúde. Entender a implementação do SUS implica entender a configuração institucional das políticas anteriores que encorajaram a expansão de redes de produção e gestão da assistência à saúde, institucionalizaram formas diversificadas de financiamento, provisão e acesso à assistência, configurando um padrão segmentado e estabelecendo-se padrões de comportamento difíceis de reverter, o que ampliaria os custos associados à adoção de alternativas diversas. Em decorrência, tiveram efeitos sobre o processo político ao modelar identidades e clivagens sociais e estruturar os interesses privados na saúde, organizados de forma a obter significativa influência nos processos decisórios subseqüentes. A inserção de categorias privilegiadas de trabalhadores em formas privadas de assistência não favoreceu a constituição de uma identidade coletiva e de valores solidaristas. Por estarem incluídos previamente em formas privadas de atenção vinculadas ao contrato de trabalho, esses segmentos, na prática, deram sustentação à assistência privada e constituíram um veto implícito à universalização da assistência pública.

A reforma que estabeleceu o sistema universal e público não logrou incorporar todos os cidadãos à assistência pública e não rompeu com o padrão segmentado. Essa duplicidade se expressa no próprio texto constitucional e se consolida no final da década de 90 com o estabelecimento 
da política regulatória, voltada para o segmento privado que formalizou essa dualidade do ponto de vista legal, normativo e institucional, paralelamente ao processo de implementação do SUS.

A falta de identificação dos segmentos organizados da população com uma assistência pública e igualitária contribuiu para o enfraquecimento da proposta de caráter publicista e universalista e para o fortalecimento da segmentação, reforçando a perspectiva de que ao SUS cabe a cobertura da população mais pobre e em condições desfavoráveis de inserção no mercado de trabalho.

Outro legado das políticas prévias é a dependência do sistema público em relação à rede privada prestadora de serviços. Ao longo das duas últimas décadas, tem ocorrido a ampliação da rede pública, principalmente a ambulatorial, que aponta tanto o esforço para universalização da assistência a partir da garantia de acesso à atenção primária e de reversão do modelo assistencial centrado no atendimento hospitalar, quanto está relacionada ao aprofundamento do processo de descentralização, gerando maior pressão sobre os governos locais.

Embora venha ocorrendo uma expansão relativa dos leitos públicos, a rede hospitalar é ainda o "calcanhar de Aquiles" do SUS e ainda é significativa a superioridade dos leitos privados: em 2005, para 0,81 leito/mil habitantes públicos, havia 1,6 privado e destes, apenas $57,6 \%$ disponíveis ao SUS (Pesquisa Assistência Médico-Sanitária - AMS. http://www.datasus.gov.br, acessado em Out/2008). Em 2002, o setor público detinha apenas $5 \%$ da rede de serviços de apoio à diagnose e terapia, formada quase que exclusivamente por estabelecimentos privados lucrativos (92\%). Entretanto, apenas 35\% desses estabelecimentos prestavam serviços para o SUS, contra $91 \%$ que vendiam serviços para os planos de saúde privados.

O fato de o sistema de saúde ser não apenas dual, mas alicerçar-se em grande parte sobre a mesma rede de serviços privados, tem implicações para o funcionamento do SUS. Em função das diferenças de valores pagos aos prestadores, caso o estabelecimento tenha condições favoráveis no mercado que lhe permitam prescindir do SUS, a sua opção será sempre vender serviços para os planos de saúde, exceto nos procedimentos para os quais os valores pagos pelo SUS sejam superiores.

\section{O que comemorar: os avanços efetivos em relação ao acesso e à integralidade - avanços internos ao SUS}

Se os legados da trajetória da política de saúde limitam a universalização da assistência pública, não são suficientes para impedir que o sistema público venha se institucionalizando. A predominância do SUS e seu impacto na população é substancial: o sistema público possui o maior número de estabelecimentos de saúde, é responsável pela maior parte dos procedimentos e pela cobertura de três quartos da população. Além disso, é no SUS que a população encontra cobertura para procedimentos tipicamente de saúde coletiva, além do fornecimento de medicamentos na atenção ambulatorial - um dos itens de maior gasto privado em saúde.

A organização da assistência foi o foco da atuação setorial desde o início da implantação do SUS e o desafio era substituir o modelo hospitalocêntrico e de livre demanda, preservando os princípios de universalidade e integralidade. Na primeira metade da década de 1990, foram criados instrumentos para a gestão pública descentralizada de estabelecimentos de saúde, homogeneizando mecanismos de pagamento e monitoramento dos serviços. Mas contando com praticamente a mesma e concentrada capacidade instalada, com a atenção centralizada no hospital e orientada para o atendimento à demanda daqueles que conseguiam acesso. $\mathrm{O}$ desafio era expandir a cobertura a fim de propiciar de fato o acesso universal, particularmente às ações de promoção e prevenção. Em 1995 foi iniciada a adoção de medidas mais efetivas para viabilização da mudança do modelo a partir das estratégias do Programa de Agentes Comunitários de Saúde (PACS) e o Programa Saúde da Família (PSF), com enfoque na promoção e prevenção, e que deveriam buscar a racionalização da utilização dos serviços, ampliar o acesso e melhorar a eficácia e a efetividade das ações, servindo de "porta de entrada" para os outros níveis do sistema de saúde - média e alta complexidades (Ministério da Saúde. http://www.saude.gov.br, acessado em Out/2008).

Entre os resultados desse esforço, a produção ambulatorial cresceu $123 \%$ no período de julho de 1994 a dezembro de 2007, sendo que os atendimentos básicos cresceram $424 \%$, ao passo que os não básicos apenas 39\%. Isso se reflete no número de internações que teve variação negativa de $16 \%$ no período de janeiro de 1995 a dezembro de 2007.

Outros indicadores sinalizam para o esforço de investimentos na atenção básica, como a redução da proporção de óbitos por diarréia, que 
passa de 10,83\% em 1990 para 4,13\% em 2005, embora com variações Regionais. Mas mesmo na Região Norte, que tem a mais alta proporção, houve redução de $12,59 \%$ para $6,45 \%$ no mesmo período.

A redução da mortalidade proporcional por causas mal definidas reflete a ampliação do acesso a serviços, tendo passado de 18,17\% em 1990 para $10,37 \%$ em 2005 , tendo maior redução na Região Norte: de $42 \%$ para $17,71 \%$ (Departamento de Informática do SUS - DATASUS. http:// www.datasus.gov.br, acessado em Out/2008).

A cobertura pelo PSF tem aumentado gradativamente, atingindo, em 2007, 95\% dos municípios, embora cobrindo apenas cerca de $50 \%$ da população e enfrentando problemas quanto à qualidade, resolutividade e fixação de recursos humanos, particularmente do médico de família. Em relação à saúde bucal, os avanços são recentes e a cobertura era de apenas $40,9 \%$ da população no mesmo ano. A implantação em 2004 do Programa Brasil Sorridente, que engloba ações com o objetivo de ampliar o acesso à saúde bucal, entre outras ações aumentou o número de equipes de saúde bucal e estas passaram a ficar vinculadas a equipes do PSF. Em 2001 havia cerca de 2.200 equipes de saúde bucal, ao passo que em 2005 este número já subira para 12.900. Mas de acordo com a Pesquisa Nacional por Amostra de Domicílios (PNAD/2003), ainda é grande a parcela de brasileiros que nunca foi ao dentista (15,9\%), embora em 1998, esta proporção fosse ainda maior $(18,7 \%)$.

A PNAD/2003 mostra outros dados significativos: para os 25,7 milhões de pessoas que buscaram atendimento de saúde nos 15 dias anteriores à pesquisa, 57,2\% dos atendimentos foram pelo SUS; a maioria da população busca atendimento em postos de saúde (79,3\%). Dados da AMS de 2002 , também apontam que $68 \%$ das internações e $73 \%$ das consultas realizadas no país foram prestadas a pacientes do SUS que é, portanto, responsável por mais de dois terços de todos os atendimentos de saúde realizados no país (Instituto Brasileiro de Geografia e Estatística - IBGE. http://www.ibge.gov.br, acessado em Out/2008).

No campo da assistência hospitalar, em setembro de 2003, o governo federal propôs a reestruturação da atenção de urgência e emergência, tendo por carro-chefe o Serviço Móvel de Atendimentos às Urgências e Emergências (SAMU), um serviço de atendimento pré-hospitalar. De acordo com o Ministério da Saúde, o SAMU está implantado em 1.136 municípios, cobrindo cerca de 100 milhões de pessoas, e tem conseguido reduzir o número de óbitos, o tempo de internação e as seqüelas decorrentes da falta de atendimento oportuno.
Da perspectiva da gestão, o SUS constitui um exemplo de pacto federativo democrático, no qual as ações são acordadas em instâncias formais com a participação das três esferas de governo e da sociedade. Destacarei aqui apenas o esforço que tem sido desenvolvido no sentido de constituir uma institucionalidade complexa para lidar com as questões relativas às condições e possibilidades de solução dos dilemas federativos no processo dinâmico de implementação da política, particularmente, a experiência recente de construção de sistemas regionalizados que envolvem articulação e cooperação entre as instâncias governamentais. A gestão da rede de serviços em contexto federativo, associada à descentralização, coloca a necessidade de combinar a autonomia dos entes federados e a cooperação entre eles de forma a garantir os preceitos constitucionais de universalidade e integralidade da atenção, compatibilizando a localização territorial das pessoas com a da rede assistencial sob a responsabilidade de gestores diversos. $\mathrm{O}$ arranjo institucional para construção de uma rede regionalizada de serviços tem avançado no sentido de resolver os problemas da coordenação, e favorecer a superação dos problemas de ação coletiva de forma a produzir uma relação cooperativa entre os entes federados, buscando-se otimizar a utilização da rede de serviços e ampliar o acesso de forma mais igualitária 4 .

\section{Desafios decorrentes de fatores exógenos ao SUS}

Se avanços consideráveis foram obtidos no âmbito do sistema de saúde, o alcance dos objetivos finalísticos do SUS está associado a questões cuja solução está fora dele, localizando-se no âmbito político da definição de políticas públicas.

O financiamento do SUS ainda é objeto de disputa, refletindo-se na instabilidade e insuficiência dos recursos alocados, colocando constrangimentos para a efetivação do SUS na sua completa acepção. A prática concreta tem negado constantemente os objetivos da reforma, expressa pelas dificuldades atuais de regulamentação da Emenda Constitucional no ${ }^{\circ} 29$ (EC29) e da não prorrogação da Contribuição Provisória sobre Movimentação Financeira (CPMF). Ao mesmo tempo, a questão do financiamento indireto da assistência supletiva por meio de incentivos ficais, tanto para pessoas jurídicas quanto físicas, não tem sido questionada na agenda pública $\mathrm{e}$ não foi discutida por ocasião da regulamentação dos planos de saúde privados. Embora a hipótese da eliminação dos subsídios necessariamente não se traduziria em aumento de recursos para o 
SUS, a permanência desse subsídio indireto para o setor privado em um quadro de insuficiência de financiamento do sistema público reafirma a dualidade da política de saúde no país.

A EC29, que fixa os percentuais mínimos a serem investidos anualmente em saúde pela União, por estados e municípios, definiu uma regra transitória para a União, que deveria ter vigorado até 2004 , mas que continua em vigor por falta de uma lei complementar que a regulamente.

A EC29 não foi capaz de garantir crescimento significativo nos recursos do governo federal para as políticas de saúde (embora tenha passado de 20 bilhões e meio em 2000 para 48 bilhões e meio em 2008) e foi parcialmente bem-sucedida na elevação dos gastos dos entes subnacionais. Ela provocou uma mudança da composição relativa do gasto público com saúde: redução relativa do gasto federal (de cerca de $60 \%$ das despesas com saúde em 2000, para pouco mais de 50\%), ampliação significativa dos gastos municipais e ampliação menor da participação dos estados no custeio da assistência. Em 2004, o gasto público per capita em saúde era assim distribuído: federal: R \$ 180,10; estadual: R\$ 180,10; municipal: R\$ 90,15 (Ministério da Saúde. http://www.saude. gov.br, acessado em Out/2008). O gasto total era de apenas $R \$ 358,54$, volume insuficiente, seja para fazer frente às necessidades do setor, seja em comparação com outros sistemas de saúde que buscam a universalidade e integralidade da atenção.

O Projeto de Lei Complementar no. 306/08, do Senado Federal, regulamenta os $\$ \S 2$ o e 30 do art. 198 da Constituição Federal de 1988 (texto da EC29, de 2000) e cria a Contribuição Social para a Saúde (CSS). O Plenário da Câmara dos Deputados aprovou em junho de 2008 essa regulamentação dos gastos com saúde e a criação da CSS por uma margem apertada de votos: 259 contra 159 (somente dois votos a mais que o mínimo necessário). Os desdobramentos dessa votação são imprevisíveis, mas não há indícios de que se obtenha suporte político para ampliação de arrecadação e, portanto, de recursos para o SUS. Além disso, a questão dos subsídios aos planos de saúde privados continua fora da agenda pública e governamental.

A questão do financiamento é apenas um dos sintomas da falta de suporte político à assistência pública, justificada, muitas vezes de forma retórica, pela avaliação negativa da qualidade dos serviços prestados pelo SUS, difundida de forma intensa na mídia e pelo setor privado. Embora muitas pesquisas, como as PNAD 1998 e 2003; pesquisa realizada pelo Instituto Brasileiro de Opinião Pública e Estatística (IBOPE) em 1998 (apud Mendes 5); pesquisa qualitativa com moradores de Belo Horizonte, realizada por Menicucci 6 em 2003, apontem avaliações positivas dos atendimentos prestados, é logicamente plausível supor que as imagens negativas, que têm como contraface a preferência pela assistência privada e reforçam a idéia de que o SUS seja voltado principalmente para os pobres, traduzam-se na falta de sustentação política do SUS, embora para a efetivação dos princípios universalistas e igualitários seria necessária a constituição de coalizões mais amplas, por se tratar de uma proposta de grande conteúdo redistributivo.

O maior desafio para uma construção institucional do sistema de saúde do Brasil que garanta maior efetividade e sustentabilidade, parece estar relacionado à definição das formas de articulação do mix privado/público que se constituiu ao longo do tempo. Dada a institucionalização de um sistema dual, isso demanda a construção social de uma agenda radicalmente inovadora que supere a discussão focada apenas no sistema "único" e possa levar a uma reconstrução do arcabouço regulatório do sistema de saúde.

A característica dual do sistema de saúde impede que o setor público tenha na rede privada a garantia da prestação de serviços públicos. Esse legado gera a necessidade de regulação governamental mais forte para, de fato, publicizar a rede privada, fazendo valer o interesse público em função do imperativo legal de garantir o acesso universal. As limitações para uma regulação mais efetiva têm sido muitas e incluem dificuldades operativas do sistema público para definir sua demanda, negociar serviços, implantar e fiscalizar contratos, o que impõe fortes custos de transação em uma relação que ainda não tem regras muito definidas e nem desenvolveu a capacidade reguladora entre os gestores públicos. As restrições políticas são também significativas, na medida em que o setor privado, com forte tradição de auto-regulação ou de regulação governamental quase que limitada à definição de preços e controle da produção dos serviços, impõe fortes resistências. Mas, o que parece mais decisivo é que não têm sido construídas, no âmbito do setor saúde ou mesmo na sociedade, propostas consistentes no sentido de uma transição para uma regulamentação sob a lógica do interesse público. Uma macrorregulação que defina a forma de inserção do setor privado no sistema de saúde não tem feito parte da agenda pública e/ou governamental e o esforço regulatório tem sido voltado para o cotidiano das operações do SUS.

É necessária também uma agenda de saúde que ultrapasse a atuação do SUS e que se sustente no conceito constitucional de direito à saúde, 
garantido por meio de um conjunto de políticas públicas. Alguns movimentos nesse sentido podem ser identificados quando a discussão de saúde tem ultrapassado o âmbito setorial, como: a discussão do aborto (que em 2006 acarretou a realização de 220 mil curetagens decorrentes); a questão do tabagismo que redundou na proibição da venda de bebidas em rodovias e na proibição de dirigir após o consumo de álcool; a 13a Conferência Nacional de Saúde/2007, que teve como tema central Saúde e Qualidade de Vida: Políticas de Estado e Desenvolvimento; a aprovação em 2006 do Pacto em Defesa do SUS, com o objetivo de consolidar a política pública de saúde como uma política de Estado e que teria no financiamento público da saúde um dos seus pilares, tendo como estratégia a mobilização social, buscando mostrar a saúde como direito de cidadania e o SUS como garantidor deste direito. Como o financiamento público é fator primordial, a mobilização se direcionaria para o alcance, em curto prazo, da regulamentação da EC29. Enfim, uma proposta de repolitizar a questão da saúde.

O lançamento do PAC-Saúde, em fevereiro de 2008, com a denominação Mais Saúde, sinaliza para a vinculação de saúde com desenvolvimento, em duas vertentes. Por um lado, retoma a discussão da saúde enquanto direito social básico, e que depende tanto da organização e do funcionamento do sistema de saúde quanto das condições gerais de vida associadas ao modelo de desenvolvimento, que contemple a inclusão social, a reversão das iniqüidades entre pessoas e regiões, o combate à pobreza, a participação e organização da sociedade. Por outro lado, trata-se de pensar a saúde como parte constitutiva da estratégia de desenvolvimento e como uma frente de expansão para um novo padrão de desenvolvimento comprometido com o bem-estar social. "A saúde contribui tanto para os direitos de cidadania quanto para a geração de investimentos, inovações, renda, emprego e receitas" (Ministério da Saúde. http://www.saude.gov.br, acessado em Out/2008).

Enfim, o alcance dos objetivos do SUS passa pela politização da questão da saúde, o que de início tem implicações para a definição do financiamento e para a resposta à questão: quanto a sociedade está disposta a pagar para o SUS? Mas que também aponta para a necessidade de uma macrorregulação que, entre outras coisas, defina a relação público/privado, além da necessidade de integração de políticas públicas não apenas voltadas para o desenvolvimento, mas também para o bem-estar. Acima de tudo, o desafio é promover uma resignificação do SUS para que ele não continue sendo o SUS para os pobres.

\section{Resumo}

O artigo faz um balanço dos avanços e desafios do Sistema Único da Saúde (SUS) a partir de uma avaliação tanto dos resultados alcançados quanto dos constrangimentos para a efetiva consolidação de seus objetivos. São enfocados três aspectos: o processo de implantação do SUS, ressaltando os constrangimentos institucionais e políticos, alguns como efeitos das políticas prévias; os avanços em relação ao acesso e à integralidade da atenção, com reflexo em alguns indicadores de saúde, e as inovações institucionais com impactos positivos na gestão do sistema; e os desafios para a consolidação dos objetivos em decorrência de fatores exógenos ao SUS, que remetem à necessidade de sua politização e maior legitimação.

Sistema Único de Saúde; Políticas Públicas de Saúde; Gestão em Saúde

\section{Referências}

1. Menicucci TMG. A implementação da reforma sanitária: a formação de uma política. Saúde Soc 2006; 15:72-87.

2. Menicucci TMG. Público e privado na política de assistência à saúde no Brasil - atores, processos e trajetória. Rio de Janeiro: Editora Fiocruz; 2007.

3. Gerschman S. A democracia inconclusa: um estudo da reforma sanitária brasileira. Rio de Janeiro: Editora Fiocruz; 1995.

4. Menicucci TMG, Marques AMF, Oliveira BR, Fortes FBCTP, Duarte VC. Regionalização da atenção à saúde em contexto federativo e suas implicações para a eqüidade de acesso e a integralidade da atenção. Belo Horizonte: Fundação João Pinheiro; 2008.

5. Mendes E. Os grandes dilemas do SUS. Salvador: Casa da Qualidade Editora; 2001.

6. Menicucci TMG. Público e privado na política de assistência à saúde no Brasil: atores, processos e trajetória [Tese de Doutorado]. Belo Horizonte: Faculdade de Filosofia e Ciências Humanas, Universidade Federal de Minas Gerais; 2003.

Recebido em 31/Mar/2009 Aprovado em 18/Mai/2009 